\title{
APPLICATION OF THE CONTROL VOLUME METHOD IN NUMERICAL MODELING OF CRYOSURGERY TREATMENT
}

\author{
Romuald Szopa ${ }^{1}$, Jarostaw Siedlecki ${ }^{2}$ \\ ${ }^{1}$ Faculty of Civil Engineering, Czestochowa University of Technology \\ ${ }^{2}$ Institute of Mathematics, Czestochowa University of Technology \\ Częstochowa, Poland \\ ${ }^{1}$ szopa@word.katowice.pl, ${ }^{2}$ jaroslawsiedlecki@gmail.com
}

\begin{abstract}
In the paper the problems of cryosurgery treatment are discussed. In particular the heat exchange between external cylindrical cryoprobe and human tissue is analyzed. The cryoprobe tip temperature changes from the initial temperature to the minimal one and back in a linear way (broken line). Thermophysical parameters determining the thermal properties of tissue can be treated as the temperature-dependent functions. The freezing model bases on the approach called 'a one domain method', and in the energy equation called a substitute thermal capacity appears. At the stage of computations the control volume method (CVM) is used. In the final part of paper the examples of computations are presented.
\end{abstract}

Keywords: bioheat transfer, modeling of cryosurgery treatment, control volume method

\section{Introduction}

The cryosurgery treatments consisting of the local freezing of tissue has recently become a popular method of treating a variety of diseases. Surface treatments on the skin tissue are performed using the so-called cylindrical cryoprobes (shape of cryoprobe tip - Fig. 1).

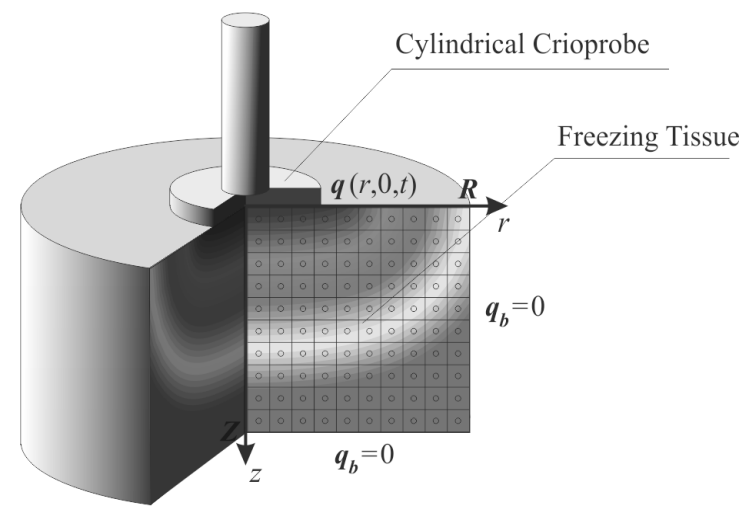

Fig. 1. Domain considered [1] 
The essential problem (from the medical point of view) is the proper choice of cryoprobe operation time ensuring the adequate depth of freezing. In particular, this time is dependent on the radius of cryoprobe tip, its operating temperature, mode of tip temperature changes and the tissue thermophysical parameters. Here, the changes of temperature from its initial value to final one $T_{\min }$ and back are assumed in a form of broken line, the cooling (heating) rates $v(-v)$ correspond to slopes of successive segments. Such a complicated problem can be solved using the numerical methods. In this paper the control volume method is used. All parameters appearing in the energy equation are assumed to be temperature-dependent.

Such a complicated problem can be solved using the numerical methods. In this paper the control volume method is used.

\section{Governing equations}

The partial differential equation (the Pennes equation $[2,3]$ ) describing the tissue freezing process can be written in the following form

$$
x \in \Omega: \quad c(T) \frac{\partial T(x, t)}{\partial t}=\operatorname{div}[\lambda(T) \operatorname{grad} T(x, t)]+Q_{p}(T)+Q_{m}(T)+L_{V} \frac{\partial f_{S}(x, t)}{\partial t}
$$

where $c$ is the tissue volumetric specific heat, $\lambda$ is the thermal conductivity, $L_{V}$ is the volumetric freezing heat, $f_{S}$ is the volumetric fraction of a frozen state at the point considered, $Q_{p}$ is the perfusion heat source, $Q_{m}$ is the metabolic heat source, $T, x, t$ denote temperature, spatial co-ordinates and time.

The perfusion heat source is equal to

$$
Q_{p}(T)=k(T)\left[T_{b}-T(x, t)\right]
$$

where $k(T)=G_{b}(T) c_{b}, \quad G_{b}$ is the tissue perfusion coefficient (dimension $\left[\mathrm{m}^{3}\right.$ blood $/ \mathrm{s} / \mathrm{m}^{3}$ tissue] $), c_{b}$ is the volumetric specific heat of blood, $T_{b}$ is the arterial blood temperature.

The fraction $f_{S}$ (see: equation (1)) is the function of temperature, that is

$$
\frac{\partial f_{S}(x, t)}{\partial t}=\frac{\mathrm{d} f_{S}(T)}{\mathrm{d} T} \frac{\partial T(x, t)}{\partial t}
$$

and then the equation (1) takes a form

$$
x \in \Omega: \quad C(T) \frac{\partial T(x, t)}{\partial t}=\operatorname{div}[\lambda(T) \operatorname{grad} T(x, t)]+Q_{p}(T)+Q_{m}(T)
$$

where

$$
C(T)=c(T)-L_{V} \frac{\mathrm{d} f_{S}(T)}{\mathrm{d} T}
$$


is the substitute thermal capacity. The introduction of this parameter leads to the model being called 'a one domain approach' or 'a fixed domain approach' $[2,3]$. If $T_{1}$ and $T_{2}$ denote the temperatures corresponding to the beginning and the end of tissue freezing then for $T>T_{1}: f_{S}(T)=0$, while for $T<T_{2}: f_{S}(T)=1$ and $C(T) \rightarrow c(T)$. So, the equation (4) describes the heat transfer processes in the whole conventionally homogenous domain. Taking into account the cryoprobe geometry the energy equation (4) should be written in the form corresponding to the cylindrical co-ordinate system $x=\{r, z\}$ (axially-symmetrical problem) meaning

$$
\begin{aligned}
& C(T) \frac{\partial T(r, z, t)}{\partial t}=\frac{1}{r} \frac{\partial}{\partial r}\left[r \lambda(T) \frac{\partial T(r, z, t)}{\partial r}\right]+ \\
& \frac{\partial}{\partial z}\left[\lambda(T) \frac{\partial T(r, z, t)}{\partial z}\right]+Q_{p}(r, z, t)+Q_{m}(r, z, t)
\end{aligned}
$$

The boundary condition given on the contact surface between tissue and cryoprobe tip is of the form of Dirichlet condition, namely

$$
x \in \Gamma_{c}: T(x, t)= \begin{cases}T_{0}-u t, & \text { if } t<t_{0}, \\ T_{\min }+u\left(t-t_{0}\right), & \text { if } t_{0} \leq t \leq 2 t_{0},\end{cases}
$$

where $T_{0}$ is the initial temperature of tissue, $u$ is the cooling (heating) rate, $t_{0}$ is the cooling time, $T_{\min }$ denotes the final temperature of a cryoprobe tip. For the others parts of external boundary (conventionally assumed cylindrical domain) the no-flux conditions are taken into account. For $t=0$ the initial temperature field is known.

In Figures 2 and 3 the courses of substitute thermal capacity and thermal conductivity of tissue are shown [1], while the other parameters are assumed to be the constant values.

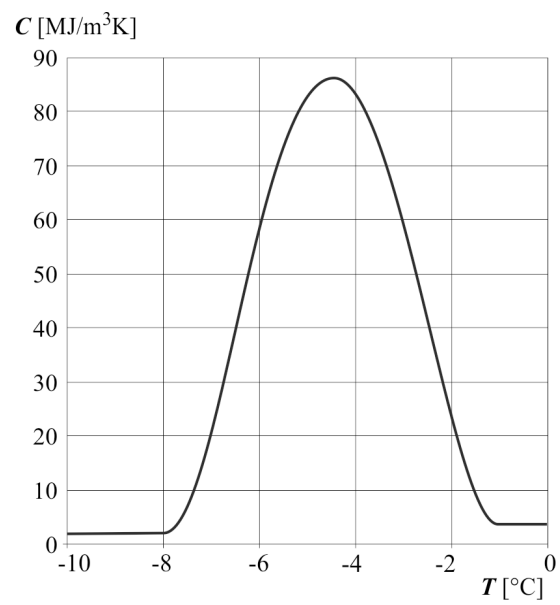

Fig. 2. Substitute thermal capacity $C(T)$ 


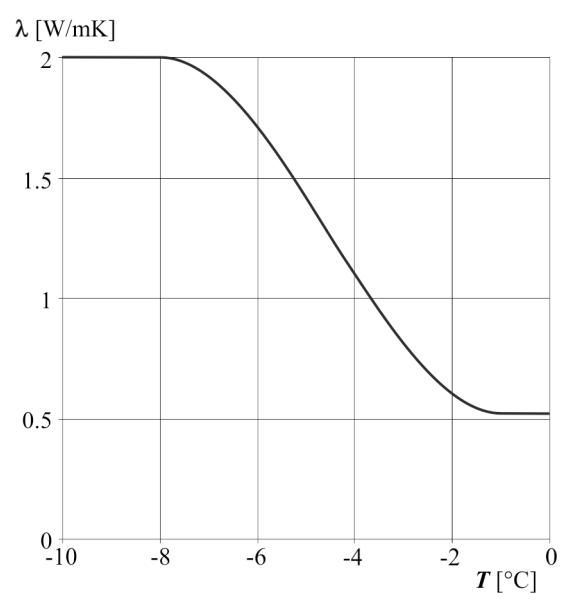

Fig. 3. Thermal conductivity

\section{The Control Volume Method}

The domain considered is divided into control volumes (CV) in the shape of rings - as in Figure 1. The central points of CV (circles) correspond to the nodes for which the time-dependent temperature field is calculated. Additionally, the time grid

$$
t^{0}<t^{1}<\ldots<t^{f-1}<t^{f}<\ldots<t^{F}<\infty
$$

with the constant time step $\Delta t$ is introduced.

For transition $t^{f-1} \rightarrow t^{f}$ the energy balance for volume $\Delta V_{0}$ can be written in the form

$$
C_{0}^{f-1}\left(T_{0}^{f}-T_{0}^{f-1}\right) \Delta V_{0}=\sum_{e=1}^{4} \frac{T_{e}^{f-1}-T_{0}^{f-1}}{R_{e}^{f-1}} \Delta A_{e} \Delta t+\left(Q_{0 p}^{f-1}+Q_{0 m}\right) \Delta V_{0} \Delta t
$$

where $e=1,2,3,4$ denotes the adjoining nodes ( 1 - right, 2 - left, 3 - top, 4 - bottom), $\Delta A_{e}$ are the surfaces limiting $\mathrm{CV}$ in $e$ direction, $R_{e}^{f-1}$ are the thermal resistances defined as follows:

$$
R_{e}^{f-1}=\frac{0.5 h_{e}}{\lambda_{0}^{f-1}}+\frac{0.5 h_{e}}{\lambda_{e}^{f-1}}
$$

where $h_{e}=\Delta r, e=1,2, h_{e}=\Delta z, e=3,4$. For the boundary nodes for which the no-flux condition is given, one should assume that in the direction to external boundary 
$R_{e}^{f-1} \rightarrow \infty$, while for the boundary with Dirichlet condition the nodal temperatures are known. Using the equation (9) one obtains

$$
T_{0}^{f}=T_{0}^{f-1}+\sum_{e=1}^{4} \frac{T_{e}^{f-1}-T_{0}^{f-1}}{R_{e}^{f-1} C_{0}^{f-1}} \Delta \Phi_{e} \Delta t+\left(Q_{0 p}^{f-1}+Q_{0 m}\right) \frac{\Delta t}{C_{0}^{f-1}}
$$

where

$$
\Phi_{1}=\frac{r_{0}+0.5 \Delta r}{r_{0} \Delta r}, \quad \Phi_{2}=\frac{r_{0}-0.5 \Delta r}{r_{0} \Delta r}, \quad \Phi_{3}=\Phi_{4}=\frac{1}{\Delta z}
$$

while $r_{0}$ is the radial co-ordinate of central node. The system of equations (11) allows one to determine 'step by step' the nodal temperatures for the successive time levels. It should be pointed out that for the explicit scheme presented, the stability condition $[4,5]$ must be respected.

\section{Results of computations}

The solution whose fragment is presented in Figure 4 has been obtained for the tip diameter equal to $10 \mathrm{~mm}$, cooling (heating) rate $u=10 \mathrm{~K} / \mathrm{min}$, cooling time $t_{0}=10 \mathrm{~min}$, the data concerning the capacities of internal heat sources and the parameters of skin tissue can be found in [5].

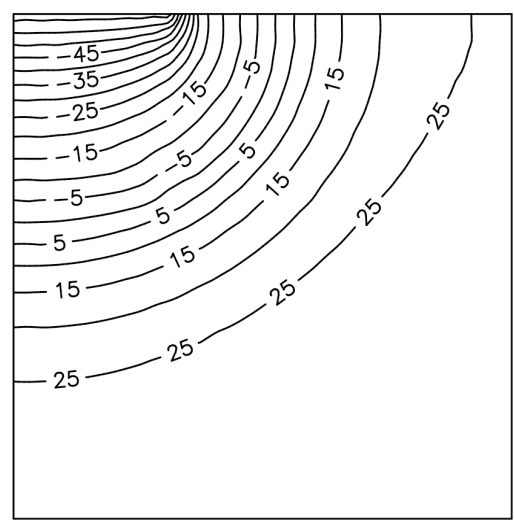

Fig. 4. Temperature field after $10 \mathrm{~min}$

\section{References}

[1] Dziewonski M., Mochnacki B., Szopa R., Sensitivity of biological tissue freezing process on the changes of cryoprobe cooling rate, Book Series: Mechanika Kaunas University of Technology 2011, 82-87. 
[2] Mochnacki B., Dziewoński M., Numerical analysis of cyclic freezing, Acta of Bioengineering and Biomechanics 2004, 6, 1, 476-479.

[3] Mochnacki B., Suchy J.S., Numerical Methods in Computations of Foundry Processes, PFTA, Cracow 1995.

[4] Majchrzak E., Mochnacki B., Numerical Methods. Theory, Practical Aspects, Algorithms, Publ. of the Silesian University of Technology, Gliwice 2004 (in Polish).

[5] Majchrzak E., Modeling and analysis of thermal phenomena, [in:] Mechanics, part 4, Vol. 12, Biomechanics, R. Bedzinski (ed.), IPPT PAN, Warsaw 2011, 223-361. 